\title{
Imaging Synaptic Protein Dynamics Using Photoactivatable Green Fluorescent Protein
}

\author{
Robby M. Weimer, Travis C. Hill, Andrew M. Hamilton, and Karen Zito
}

\begin{abstract}
Considerable evidence has accumulated that structural changes in dendritic spines and their synapses are associated with adaptive functional changes in cortical circuits, such as during circuit refinement in young animals and in learning and memory in adults. Understanding the mechanisms of circuit plasticity requires detailed investigation of the structural dynamics of dendritic spines and how they are regulated by neural activity and sensory experience. Studying the dynamic localization of synaptic proteins in dendritic spines and how their stabilization and exchange rates influence spine structural plasticity is also important. This protocol describes imaging approaches to study synaptic protein dynamics in dendritic spines of the rodent cerebral cortex. It gives a strategy for generating photoactivatable green fluorescent protein (PA-GFP)-tagged synaptic proteins and in vitro and in vivo transfection methods for coexpression of these proteins with a spectrally separable cell-filling marker (DsRed-Express). Methods for tracking synaptic protein localization using photoactivation and time-lapse imaging of PA-GFP in spiny pyramidal neuron dendrites are given. A discussion of imaging hardware and software preferences is also included. The methods described here can be used to study the dynamic processes underlying spine synapse development during the formation and plasticity of neural circuits in the mammalian brain.
\end{abstract}

It is essential that you consult the appropriate Material Safety Data Sheets and your institution's Environmental Health and Safety Office for proper handling of equipment and hazardous materials used in this protocol.

RECIPE: Please see the end of this article for recipes indicated by $<R>$. Additional recipes can be found online at http://cshprotocols.cshlp.org/site/recipes.

Reagents

aCSF for hippocampus $(\sim 40 \mathrm{~mL})<\mathrm{R}>$

Expression vectors, PA-GFP and DsRed-Express

Hippocampal slice cultures, organotypic, prepared 1-2 wk before in vitro imaging is planned (see Step 3)

Alternatively, use pregnant female mice if in vivo imaging will be performed.

Reagents for testing PA-GFP-tagged synaptic proteins for their ability to retain native protein function (see Step 2)

Adapted from Imaging in Developmental Biology (ed. Sharpe and Wong). CSHL Press, Cold Spring Harbor, NY, USA, 2011. 
R.M. Weimer et al.

Equipment

Biolistic system, handheld (e.g., Bio-Rad Laboratories 165-2431 or equivalent) (for tranfecting hippocampal slice cultures; see Step 3)

Alternatively, use an electroporation system for in utero electroporation in mice.

Equipment for testing PA-GFP-tagged synaptic proteins for their ability to retain native protein function (see Step 2)

Filter sets for dual color imaging (Chroma Technology)

Imaging setup:

- When imaging in vivo or deep in tissue slices, two-photon microscopy provides distinct advantages, primarily by reducing background fluorescence and minimizing phototoxicity (Svoboda and Yasuda 2006). Two-photon microscopes suitable for imaging dendritic spines can be custom-built (e.g., Mainen et al. 1999; Majewska et al. 2000) or obtained from a commercial vendor (e.g., Olympus, Prairie Technologies, Sutter Instruments, Zeiss). A typical custom imaging setup for two-photon imaging of dendritic spines is shown in Figure 1. The power of the infrared (IR) Ti::sapphire laser (e.g., Coherent or Spectra-Physics) beam is controlled with a Pockels cell (e.g., Conoptics 350-80LA) and $x-$ $y$ positioning is controlled with galvanometers (e.g., Cambridge Technology 6210). The beam is directed through a scan lens into a microscope head and focused through a waterimmersion objective that is designed to readily transmit IR light (typically $20 \times-60 \times, 0.8-$ 0.9 numerical aperture [NA]; e.g., Olympus LUMPLFLN 60XW/IR2). For in vivo imaging, it is best if the microscope is mounted on an independently translatable $x-y$ stage. Emitted photons are collected with photomultiplier tubes (PMTs; e.g., Hamamatsu R3896), which should be mounted as close to the preparation as possible to reduce loss of signal. For in vitro imaging, it is desirable to install a second set of PMTs below the condenser (single asterisks in Fig. 1).

- A second pulsed Ti::sapphire laser (double asterisks in Fig. 1) is usually required for simultaneous two-photon imaging and photoactivation. This second laser can be combined with the first beam upstream of the scan mirrors and scanned simultaneously, or scanned independently and combined downstream from two independent sets of scan mirrors. Alignment of the imaging and photoactivation lasers should be regularly monitored and optimized.

- Software that permits automatic stage control and looping through multiple image acquisition positions is useful for time-lapse imaging in vivo or in vitro, so that multiple dendrites can be relocated rapidly and imaged at each time point. The open source MATLAB software ScanImage is a highly configurable software suitable for two-photon imaging experiments (Pologruto et al. 2003; http://research.janelia.org/labs/display/ephus/).

Subresolution fluorescent beads, yellow-green fluorescence, 0.1- $\mu \mathrm{m}$ (FluoSpheres; Invitrogen F-8803)

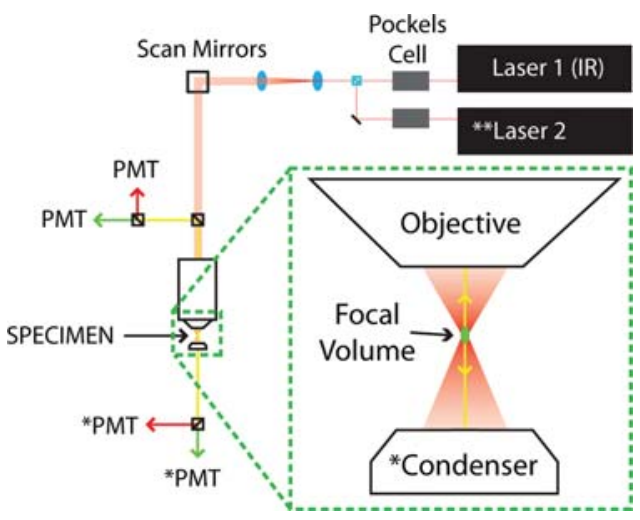

FIGURE 1. Diagram of setup for simultaneous two-photon imaging and photostimulation. For a typical two-photon imaging microscope, the power of the pulsed IR lasers is controlled with Pockels cells, and $x-y$ positioning is controlled with scan mirrors. Two-photon excitation is primarily restricted to the focal volume, resulting in localization of excitation (inset, green dot). For both in vivo and in vitro imaging, emitted photons are collected by PMTs above the sample. A condenser and bottom PMTs (single asterisks) are used for in vitro imaging to maximize the collection of emitted photons. A second laser (double asterisks) is typically required for photoactivation experiments. 


\section{Preparation of PA-GFP-Tagged Synaptic Proteins}

1. Clone the PA-GFP cDNA (Patterson and Lippincott-Schwartz 2002) in frame with either the $5^{\prime}$ or $3^{\prime}$ end of the synaptic protein coding sequence under the control of the CAGGS promoter (Niwa et al. 1991).

2. Test PA-GFP-tagged synaptic proteins for their ability to retain native protein function by assessing protein localization pattern, ability to bind to known partners, and/or ability to complement a knockout or knockdown of endogenous protein levels.

\section{Neuronal Transfection for In Vitro and In Vivo Imaging Preparations}

3. Transfect the samples of choice with the construct.

\section{Cultured Slice Preparations}

i. Using biolistic transfection, cotransfect organotypic hippocampal slice cultures with unattached PA-GFP or the PA-GFP-tagged synaptic protein and DsRed-Express 2-3 d before imaging.

An online video protocol is available for biolistic transfection of hippocampal slice cultures (Woods and Zito 2008).

ii. Image transfected pyramidal neurons in aCSF for hippocampus using a two-photon microscope.

\section{In Vivo Imaging Preparations}

i. Cotransfect unattached PA-GFP or the PA-GFP-tagged synaptic protein and DsRed-Express using in utero electroporation in mice (Saito 2006).

ii. Install a chronic cranial imaging window $2 \mathrm{wk}$ before imaging in adult mice and $2 \mathrm{~d}$ before imaging in neonatal mice.

A protocol for installing a chronic cranial imaging window for in vivo imaging is detailed in Holtmaat et al. (2011).

\section{Choosing Photoactivation and Imaging Wavelengths}

Because photoactivation and imaging of photoactivated PA-GFP require different wavelengths of light, photoactivation experiments are best conducted with two lasers. Align photoactivation and imaging lasers on a daily basis using subresolution fluorescent beads.

4. Use a single laser to image both green and red fluorophores.

GFP, photoactivated PA-GFP, and DsRed-Express can be efficiently excited with wavelengths ranging from 910 to $1020 \mathrm{~nm}$. Choose an imaging wavelength that does not photoactivate PA-GFP. It is important to confirm that there is no increase in green fluorescence during time-lapse imaging of PA-GFP-tagged proteins in the absence of photoactivation. We typically use 930, 980, or $1020 \mathrm{~nm}$ for imaging photoactivated PA-GFP on a two-photon microscope.

5. Determine the optimal photoactivation wavelength for each PA-GFP-tagged synaptic protein by measuring the percent increase in fluorescence in response to photoactivation pulses of different wavelengths, while holding constant the pulse power and duration.

Unattached PA-GFP is efficiently photoactivated at $810 \mathrm{~nm}$ (Fig. 2A). However, PA-GFP-tagged proteins can vary in their preferred photoactivation wavelength (A. Hamilton and K. Zito, unpubl.).

\section{Photoactivation}

6. Identify a target spine based on the cell-filling marker, DsRed-Express (Fig. 2A).

To ensure that the photoactivation pulse will not activate neighboring spines or the dendrite, choose target spines that are well separated $(>1 \mu \mathrm{m})$ from neighboring spines and with spine heads that are well separated from the dendrite. 
R.M. Weimer et al.

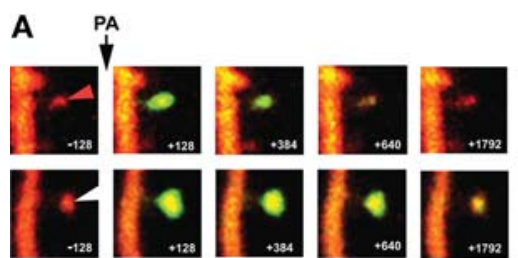

C

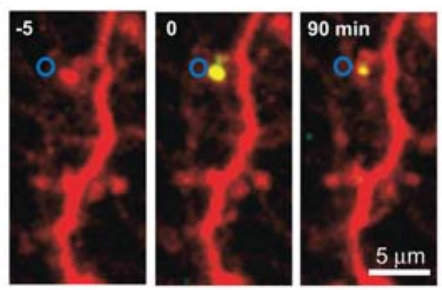

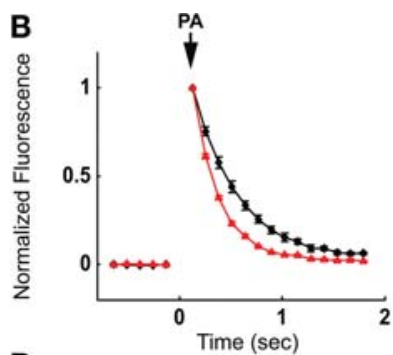

D

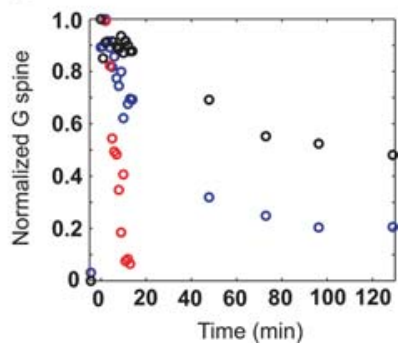

FIGURE 2. Photoactivation of PA-GFP and PA-GFP-tagged synaptic proteins. (A) Images collected before and after photoactivation (PA) of PA-GFP in vitro at $810 \mathrm{~nm}$ for two spines showing overlay of DsRed-Express (red) and PA-GFP (green) fluorescence. Overlap of green and red fluorescence appears yellow. Time stamps are in milliseconds. $(B)$ Time course of PA-GFP fluorescence decay after photoactivation (PA) for two spines from $A$. Data points following photoactivation are fit to a single exponential to extract the time constant of equilibration. (Adapted, with permission of Elsevier, from Zito et al. 2009.) (C) Dendrites of neurons transfected in utero with PSD-95-PA-GFP (green) and DsRed-Express (red) were imaged in vivo through a cranial window at postnatal day 12 (P12). One spine (blue circle) was targeted for photoactivation. $(D)$ Decay of PSD-95-PA-GFP fluorescence (normalized to the first time point after photoactivation) reveals the time course of PSD-95 exchange between the spine and dendrite at P12 (blue circles), at P66 (black circles), and after mutations in two protein interaction domains (red circles). (R.M. Weimer, N. Gray, and K. Svoboda, unpubl.)

7. If possible, locate multiple control spines within the field of view containing the target spine.

8. Use a field of view of $\sim 18-25 \mu \mathrm{m}$ to image spines before photoactivation.

9. Illuminate a box $(\sim 1.5 \times 1.5 \mu \mathrm{m})$ centered on the spine head with a pulsed Ti::sapphire laser tuned to the photoactivation wavelength (typically $810 \mathrm{~nm}$ for unattached PA-GFP).

Photoactivation can be facilitated by zooming in to use a field of view of $\sim 5 \mu \mathrm{m}$ during the photoactivation trial.

10. To optimize the signal while at the same time minimizing phototoxicity, choose photoactivation powers that consistently increase green fluorescence levels by at least two standard deviations above the mean baseline fluorescence. For unattached PA-GFP in spines at depths of 20-60 $\mu \mathrm{m}$, we typically use powers in the objective back-focal plane of $80-100 \mathrm{~mW}$ in vitro and $125 \mathrm{~mW}$ in vivo.

For PA-GFP-tagged proteins, which are usually less abundant and less dynamic than unattached PA-GFP, photoactivation often is best achieved at lower powers (45-65 $\mathrm{mW}$ in vitro and 80-100 $\mathrm{mW}$ in vivo) than those used for unattached PA-GFP to avoid excessive photobleaching of the photoactivated PA-GFP-tagged protein.

Time-Lapse Imaging of Fluorescence Decay

11. Following photoactivation, use time-lapse imaging to monitor decay of activated PA-GFP fluorescence.

Photoactivated PA-GFP and DsRed-Express fluorescence emissions can be effectively separated using a 565-nm dichroic mirror and barrier filters centered at 525 and $600 \mathrm{~nm}$, respectively.

- For unattached PA-GFP (Fig. 2A), a typical photoactivation trial consists of 25 continuous frames $(64 \times 64$ pixels, $128 \mathrm{msec}$ per frame): 10 frames of baseline, one photoactivation frame, and 14 frames following photoactivation (Zito et al. 2009). Usually, five to six trials per spine are performed at 10 -sec intervals.

Alternatively, use line scans to monitor photoactivated PA-GFP fluorescence decay at higher temporal resolution, typically at 2 msec per line (Bloodgood and Sabatini 2005). 
Analysis

- For PA-GFP-tagged proteins (e.g., PSD-95-PA-GFP; Fig. 2C), a typical photoactivation trial consists of 16 time points: one time point before photoactivation, one photoactivation time point, and 10 time points collected at $\sim 90$-sec intervals after photoactivation (to capture the initial fast decay component) followed by four time points collected at $\sim 20$ min intervals (Gray et al. 2006). Because sample drift can occur over these longer time intervals, it is important to acquire image stacks (10-20 frames, $256 \times 256$ pixels, $500 \mathrm{msec}$ per frame) instead of single image frames at each time point.

The image acquisition frequency and trial duration will depend on the rate of exchange of the specific PA-GFP-tagged protein. In general, sample $\sim 10$ time points during the initial $10 \%$ decay in fluorescence and then decrease image acquisition frequency to once every 20 min to minimize photobleaching.

12. Measure total green fluorescence decay over time by integrating the green channel pixel intensity within a region of interest surrounding the spine head for each frame or line in the time series.

- For unattached PA-GFP (Fig. 2B), calculate the time constant of green fluorescence decay by fitting the green fluorescence signal after photoactivation with a single exponential (Bloodgood and Sabatini 2005).

Spine morphology, spine age, and neuronal activity can influence diffusional coupling between spine and dendrite (Bloodgood and Sabatini 2005; Gray et al. 2006; Zito et al. 2009). Therefore, care should be taken to carefully control for these variables when comparing populations of spines under different experimental conditions.

- For PA-GFP-tagged proteins (e.g., PSD-95-PA-GFP; Fig. 2D), quantitatively assess protein exchange by measuring the proportion of fluorescence decay over a given period of time (Xu et al. 2008). (This method provides a relatively straightforward means to compare decay rates between various experimental conditions without making assumptions regarding the biophysical parameters governing the exchange.)

Alternatively, estimate a time constant for each component of the decay curve (Gray et al. 2006). (This method can provide more detailed estimates of the kinetics underlying exchange, such as binding constants. Resolving and properly fitting multiple time constants can be challenging, often requiring computer modeling.)

The cellular and molecular interactions underlying localization and stability of synaptic proteins within a spine can be studied by measuring the rate of synaptic protein exchange between individual spines and the dendrite. Two approaches have been widely adopted: fluorescence recovery after photobleaching (FRAP) and photoactivation. In FRAP experiments (Zito et al. 2004; Kuriu et al. 2006; Sharma et al. 2006; Tsuriel et al. 2006), the spine is targeted for increased illumination at the excitation wavelength to photobleach a fluorescently tagged synaptic protein. As bleached proteins exchange with unbleached proteins, fluorescence intensities increase within the bleached spine. Conversely, in photoactivation paradigms (Gray et al. 2006; Tsuriel et al. 2006; Xu et al. 2008; Sturgill et al. 2009), spines are illuminated with light at a wavelength that will convert a protein tagged with a photoactivatable fluorophore, such as PA-GFP (Patterson and Lippincott-Schwartz 2002), from a dim to a bright state. As photoactivated proteins exchange with nonphotoactivated proteins, fluorescence intensities decrease within the spine. Although FRAP and photoactivation paradigms can provide similar types of data, photoactivation approaches are particularly advantageous because they can provide greater specificity and additional temporal information concerning spatial localization as only the photoactivated protein population is tracked.

Choosing a Preparation: In Vitro or In Vivo?

Studies of dendritic spines and spine synapses are best performed in preparations that preserve the local environment, preferably in vivo, or deep within acute or cultured brain slices. In vitro 
R.M. Weimer et al.

preparations are advantageous for experiments combining imaging and electrophysiology of transfected neurons, for rapid pharmacological manipulations and photolysis of caged compounds, and for imaging low-abundance fluorescently tagged proteins, which can be difficult to detect in vivo. Virtually any brain region is amenable for acute or cultured slice preparation; some structures, like the hippocampus, are particularly robust in slice culture. Cultured slices are usually maintained for at most four weeks, limiting chronic time-lapse experiments to this time scale. Application of time-lapse imaging in vitro is relatively straightforward, primarily involving assembling temperature control and perfusion systems on a two-photon microscope to image spines under physiological conditions.

In vivo approaches are advantageous for imaging dendritic spines and synaptic proteins in their native environment and also for studying changes in spines and synapses that occur in response to sensory or behavioral manipulations. Time-lapse measurements in vivo can span weeks and even months to years. Most commonly, in vivo studies comprise either acute time spans of a few hours or chronic time spans involving one imaging session every few days (or longer intervals). In vivo imaging of dendritic spines is most often accomplished through a chronically implanted "window" (Trachtenberg et al. 2002) or a temporarily "thinned" skull (Grutzendler et al. 2002). Spines can most readily be imaged in cortical layers 1 and 2/3. Structures buried deeper in the brain, such as the hippocampus, are accessible only after extensive surgery to remove most of the cortex (Mizrahi et al. 2004) or through specialized endoscopic approaches (Barretto et al. 2009). Thus, spine development in these deep brain structures has rarely been studied in vivo.

Uses of the Method

Time-lapse two-photon imaging in vitro and in vivo has led to many discoveries concerning the dynamic morphological plasticity of dendritic spines during development and in the adult brain. Further advances have been realized by combining time-lapse imaging with dual-color imaging, FRAP, and photoactivation to monitor the localization and dynamics of fluorescently tagged synaptic proteins. Measuring exchange of a synaptic protein between spine and dendrite has provided estimates of relative synaptic protein stability and binding/unbinding rates for synaptic proteins with their binding partners. When combined with structure-function approaches, the precise site of individual molecular interactions can be probed (Zito et al. 2004; Xu et al. 2008; Sturgill et al. 2009). When combined with behavioral and pharmacological manipulations and time-lapse imaging of spine dynamics, the relationship between synaptic activity, synaptic protein exchange rates, and spine stability can be assessed (Gray et al. 2006; Kuriu et al. 2006; Sharma et al. 2006; Tsuriel et al. 2006; Sturgill et al. 2009). The discoveries made using these powerful techniques will ultimately contribute to unraveling the intricate mechanisms involved in the formation and maturation of dendritic spines and their synapses.

\section{RECIPE}

aCSF for Hippocampus

\begin{tabular}{lcc} 
Reagent & Amount to add (for 2 L) & Final concentration \\
\hline $\mathrm{NaCl}$ & $14.84 \mathrm{~g}$ & $127 \mathrm{~mm}$ \\
$\mathrm{NaHCO}_{3}$ & $4.2 \mathrm{~g}$ & $25 \mathrm{mM}$ \\
$\mathrm{NaH}_{2} \mathrm{PO}_{4} \cdot \mathrm{H}_{2} \mathrm{O}$ & $0.35 \mathrm{~g}$ & $1.25 \mathrm{~mm}$ \\
$\mathrm{KCl}$ & $0.37 \mathrm{~g}$ & $2.5 \mathrm{~mm}$ \\
D-glucose & $9.01 \mathrm{~g}$ & $25 \mathrm{~mm}$ \\
$1 \mathrm{M} \mathrm{MgCl}$ & $2 \mathrm{~mL}$ & $1 \mathrm{mM}$ \\
$1 \mathrm{M} \mathrm{CaCl}$ & $4 \mathrm{~mL}$ & $2 \mathrm{mM}$ \\
$\mathrm{H}_{2} \mathrm{O}$ & to $2 \mathrm{~L}$ & -
\end{tabular}

Bubble with carbogen $\left(95 \% \mathrm{O}_{2} / 5 \% \mathrm{CO}_{2}\right)$ for $15-20 \mathrm{~min}$. Add the $\mathrm{CaCl}_{2}$ only after bubbling with carbogen. Adjust the $\mathrm{pH}$ to $7.25-7.35$ with $\mathrm{NaOH}$. Check the osmolarity; it should be $\sim 310-320 \mathrm{mOsm}$. Store at $4^{\circ} \mathrm{C}$. 
Thanks to Linda Wilbrecht, Georgia Woods, and Lauren Boudewyn for discussions and critical reading of the manuscript. This work was supported by the Hellman Family Foundation (K.Z.), the National Science Foundation (K.Z.), the National Institutes of Health (NINDS R01-NS062736 to K.Z., NIDCD T32-DC008072 to T.C.H., NIGMS T32-GM007377 to A.M.H.), and a Burroughs Wellcome Fund Career Award in the Biomedical Sciences (K.Z.).

\section{REFERENCES}

Barretto RP, Messerschmidt B, Schnitzer MJ. 2009. In vivo fluorescence imaging with high-resolution microlenses. Nat Methods 6: 511-512.

Bloodgood BL, Sabatini BL. 2005. Neuronal activity regulates diffusion across the neck of dendritic spines. Science 310: 866-869.

Gray NW, Weimer RM, Bureau I, Svoboda K. 2006. Rapid redistribution of synaptic PSD-95 in the neocortex in vivo. PLoS Biol 4: e370. doi: 10.1371/journalpbio.0040370.

Grutzendler J, Kasthuri N, Gan W-B. 2002. Long-term dendritic spine stability in the adult cortex. Nature 420: 812-816.

Holtmaat A, de Paola V, Wilbrecht L, Trachtenberg J, Svoboda K, PorteraCailliau C. 2011. Imaging neocortical neurons through a chronic cranial window. In: Imaging in neuroscience: A laboratory manual (ed F Helmchen, A Konnerth). Cold Spring Harbor Laboratory Press, Cold Spring Harbor, NY (in press).

Kuriu T, Inoue A, Bito H, Sobue K, Okabe S. 2006. Differential control of postsynaptic density scaffolds via actin-dependent and -independent mechanisms. J Neurosci 26: 7693-7706.

Mainen ZF, Maletic-Savatic M, Shi SH, Hayashi Y, Malinow R, Svoboda K. 1999. Two-photon imaging in living brain slices. Methods 18: 231-239.

Majewska A, Yiu G, Yuste R. 2000. A custom-made two-photon microscope and deconvolution system. Pflugers Arch 441: 398-408.

Mizrahi A, Crowley JC, Shtoyerman E, Katz LC. 2004. High-resolution in vivo imaging of hippocampal dendrites and spines. J Neurosci 24: 3147-3151.

Niwa H, Yamamura K, Miyazaki J. 1991. Efficient selection for high-expression transfectants with a novel eukaryotic vector. Gene 108: 193-199.

Patterson GH, Lippincott-Schwartz J. 2002. A photoactivatable GFP for selective photolabeling of proteins and cells. Science 297: 1873-1877.

Pologruto TA, Sabatini BL, Svoboda K. 2003. ScanImage: Flexible software for operating laser scanning microscopes. Biomed Eng Online 2: 13. doi: $10.1186 / 1475-925 X-2-13$.
Saito T. 2006. In vivo electroporation in the embryonic mouse central nervous system. Nat Protoc 1: 1552-1558.

Sharma K, Fong DK, Craig AM. 2006. Postsynaptic protein mobility in dendritic spines: Long-term regulation by synaptic NMDA receptor activation. Mol Cell Neurosci 31: 702-712.

Sturgill JF, Steiner P, Czervionke BL, Sabatini BL. 2009. Distinct domains within PSD-95 mediate synaptic incorporation, stabilization, and activity-dependent trafficking. J Neurosci 29: 12845-12854.

Svoboda K, Yasuda R. 2006. Principles of two-photon excitation microscopy and its applications to neuroscience. Neuron 50: 823-839.

Trachtenberg JT, Chen BE, Knott GW, Feng G, Sanes JR, Welker E, Svoboda K. 2002. Long-term in vivo imaging of experience-dependent synaptic plasticity in adult cortex. Nature 420: 788-794.

Tsuriel S, Geva R, Zamorano P, Dresbach T, Boeckers T, Gundelfinger ED, Garner CC, Ziv NE. 2006. Local sharing as a predominant determinant of synaptic matrix molecular dynamics. PLoS Biol 4: e271. doi: 10.1371/ journal. pbio.0040271.

Woods G, Zito K. 2008. Preparation of gene gun bullets and biolistic transfection of neurons in slice culture. J Vis Exp 12. http://www.jove.com/ index/Details.stp?ID=675, doi: 10.3791/675.

Xu W, Schlüter OM, Steiner P, Czervionke BL, Sabatini B, Malenka RC. 2008. Molecular dissociation of the role of PSD-95 in regulating synaptic strength and LTD. Neuron 57: 248-262.

Zito K, Knott G, Shepherd GM, Shenolikar S, Svoboda K. 2004. Induction of spine growth and synapse formation by regulation of the spine actin cytoskeleton. Neuron 44: 321-334.

Zito K, Scheuss V, Knott G, Hill T, Svoboda K. 2009. Rapid functional maturation of nascent dendritic spines. Neuron 61: 247-258. 


\section{Imaging Synaptic Protein Dynamics Using Photoactivatable Green Fluorescent Protein}

Robby M. Weimer, Travis C. Hill, Andrew M. Hamilton and Karen Zito

Cold Spring Harb Protoc; doi: 10.1101/pdb.prot070029

\begin{tabular}{|c|c|}
\hline $\begin{array}{l}\text { Email Alerting } \\
\text { Service }\end{array}$ & Receive free email alerts when new articles cite this article - click here. \\
\hline $\begin{array}{l}\text { Subject } \\
\text { Categories }\end{array}$ & $\begin{array}{l}\text { Browse articles on similar topics from Cold Spring Harbor Protocols. } \\
\text { Cell Imaging (525 articles) } \\
\text { Fluorescent Proteins ( } 259 \text { articles) } \\
\text { Image Analysis ( } 124 \text { articles) } \\
\text { Imaging for Neuroscience ( } 342 \text { articles) } \\
\text { In Vivo Imaging ( } 334 \text { articles) } \\
\text { Mouse (437 articles) } \\
\text { Multi-Photon Microscopy (103 articles) } \\
\text { Photoactivation ( } 37 \text { articles) } \\
\text { Video Imaging / Time Lapse Imaging (171 articles) } \\
\text { Visualization of Proteins (107 articles) }\end{array}$ \\
\hline
\end{tabular}

